\title{
ON MONOGENIC OPERATORS AND MEASURES
}

\author{
Z. LIPECKI AND D. PLACHKY
}

\begin{abstract}
The notion of a monogenic operator between linear lattices, generalizing that of a monogenic measure, is introduced and investigated. The decomposition of an operator into its monogenic and antimonogenic parts is established. Products of monogenic measures are also considered.
\end{abstract}

Introduction. We introduce the notion of a monogenic operator between linear lattices, i.e. of an order bounded linear operator which is determined in some sense by its restriction to a given linear sublattice. We prove that monogenic operators form a band (Theorem 1). This yields the decomposition of an arbitrary operator into its monogenic and antimonogenic parts. In a special case where operators are identified with real-valued measures on a $\sigma$-field we get a generalization of a result of Johnson [4]. We compare our decomposition with some classical decompositions in measure theory (Examples 1 and 2). We also consider (not necessarily direct) products of monogenic measures (Theorem 2 and Example 3). As far as the linear-lattice-theoretical terminology is concerned we mostly follow Jameson's book [3].

1. Monogenic operators. Let $X$ and $Y$ be real linear lattices and assume that $Y$ is order complete. Then the set $L_{b}(X, Y)$ of order bounded linear operators from $X$ into $Y$ is an order complete linear lattice [3, Theorem 2.6.1]. We denote by $L_{+}(X, Y)$ its subset consisting of all positive (i.e. monotonic in the terminology of [3]) operators. Let $M$ be a cofinal (i.e. majorizing) linear sublattice of $X$ and $\mathcal{G}$ be a solid subspace of $L_{b}(X, Y)$. We say that $T \in \mathcal{G}$ is monogenic (with respect to $M$ and $\mathcal{G}$ ) provided $|T| M|=| T|| M$ and there is no other $S \in \mathcal{G}$ with this property which coincides with $T$ on $M$ (more precisely, $|S| M|=| S|| M$ and $S|M=T| M$ imply $S=T$ ). In case $T$ is positive, this definition can be made simpler. Moreover, alternate descriptions of monogenicity are available. ${ }^{1}$ Namely we have

LEMMA 1. Suppose $T \in \mathcal{G}_{+}$. Then the following four conditions are equivalent:

(i) $T$ is monogenic.

(ii) For any $S \in g_{+}$with $S|M=T| M$ we have $S=T$.

(iii) For any $S \in \mathcal{G}_{+}$with $S|M<T| M$ we have $S<T$.

(iv) For any $S \in g_{+}$with $S|M>T| M$ we have $S>T$.

Received by the editors June 15, 1979.

AMS (MOS) subject classifications (1970). Primary 28A10, 28A35, 47B55.

Key words and phrases. Linear lattice, band, monogenic operator, monogenic measure, products of measures.

${ }^{1}$ Note that for $g=L_{b}(X, Y)$ an intrinsic characterization of positive monogenic operators is possible (see [5, Theorem 1]). 0002-9939/81/0000-0261/\$02.25 
Proof. Clearly, (i) $\Rightarrow$ (ii). To prove the converse implication, it is enough to show that if $S \in L_{b}(X, Y)$ and $S|M=| S|| M$, then $S$ is positive. Let $x \in X_{+}$and take $z \in M$ with $x<z$. Then $|x-z|<z$. It follows that $S(x)=|S|(z)+S(x-z)>$ 0 . Next we prove that (ii) $\Rightarrow$ (iii). Let $S$ satisfy the assumptions of (iii). By Theorem 2.6.3 in [3] applied to the lattice-monotonic sublinear mapping $P(x)=T(|x|)$, there exists $S_{1} \in L_{+}(X, Y)$ with $S_{1}|M=S| M$ and $S_{1}<T$. Hence $\left(\left(T-S_{1}\right)+S\right) \mid M=$ $T \mid M$ and $T-S_{1}+S \in \mathcal{G}_{+}$, so that in view of (ii), $S=S_{1}$, and we are done. A similar, but simpler argument yields (ii) $\Rightarrow$ (iv). Finally the implications (iii) $\Rightarrow$ (ii) and (iv) $\Rightarrow$ (ii) are an easy consequence of the following

LemMa 2. If $S, T \in L_{+}(X, Y), S<T$ and $S|M=T| M$, then $S=T$.

Proof. Given $x \in X$ choose $z \in M$ with $x<z$. Then $S(x)=S(z)-S(z-x)$ $=T(z)-S(z-x)>T(z)-T(z-x)=T(x)$.

THEOREM 1. The set of monogenic operators is a band in $g^{2}$

Proof. First we show that if $T \in \mathcal{G}$ is monogenic, then so is $|T|$. Take $W \in \mathcal{G}_{+}$ with $|T||M=W| M$. In view of Lemmas 1 and 2 , it is enough to show that $|T|<W$. By the generalized version of the Hahn-Banach theorem [3, 2.5.7], there exists a linear operator $T_{1}: X \rightarrow Y$ with $T_{1}|M=T| M$ and $\left|T_{1}(x)\right|<W(|x|)$ for $x \in X$. Hence $\left|T_{1}\right|<W$ and $T_{1} \in \mathcal{G}$. As $T$ is monogenic, we have $\left|T_{1} \| M<W\right| M$ $=|T| M|=| T_{1}|M|$, and so $T_{1}=T$. Thus $|T|<W$.

In order to establish the converse assertion suppose $T \in \mathcal{g}$ and $|T|$ is monogenic, and take $S \in \mathcal{G}$ with $|S| M|=| S|| M$ and $S|M=T| M$. Then $|S| \mid M<$ $|T| \mid M$, whence by Lemma $1,|S|<|T|$. It follows that for any $x \in X$ and $z \in Z$ we have $|S(x)-T(x)|=|S(x-z)-T(x-z)|<2|T|(|x-z|)$. Hence, in view of Theorem 3 in [5], $S(x)=T(x)$.

Suppose $T_{1}, T_{2} \in \mathcal{G}_{+}$are monogenic. We claim that $T_{1}+T_{2}$ is also monogenic. According to Lemma 1 , it is enough to show that given $S \in g_{+}$with $\left(T_{1}+T_{2}\right)|M<S| M$ we have $T_{1}+T_{2}<S$. The latter follows easily by a repeated application of Lemma 1.

Clearly, $t T$ is monogenic for any $T \in \mathcal{G}$ and $t \in \mathbf{R}$. It is also easily seen from Lemma 1 that if $T \in \mathscr{g}_{+}$is monogenic, then so is any $S \in L_{+}(X, Y)$, with $S<T$. These observations together with what we have proved so far show that the set of monogenic operators is a solid subspace of $g$. Thus in order to prove that it is a band, it is enough to note that if $T_{\alpha} \in \mathcal{G}_{+}$are monogenic and $T=\sup T_{\alpha}$ (in $\mathscr{g}$ ), then $T$ is also monogenic [3, 2.4.3]. But this is so as $T$ satisfies condition (iv) of Lemma 1.

We say that $T \in \mathcal{G}$ is antimonogenic (with respect to $M$ and $\mathcal{G}$ ) provided $T$ is disjoint from all monogenic operators in $g$. Theorem 1 and the Riesz decomposition theorem $[3,2.5 .4]$ yield the following

\footnotetext{
${ }^{2}$ Dr. S. S. Kutateladze has kindly informed us that a result related to Theorem 1 can be found in his paper Choquet boundaries in K-spaces, Uspehi Mat. Nauk 30 (1975), 107-146, Theorem 3.1; English transl. in Russian Math. Surveys 30 (1975), 115-155.
} 
Corollary 1. Suppose $g$ is a band in $L_{b}(X, Y)$. Then each $T \in \mathcal{g}$ can be uniquely decomposed in the following way: $T=T_{1}+T_{2}$, where $T_{1}, T_{2} \in \mathcal{G}, T_{1}$ is monogenic and $T_{2}$ is antimonogenic.

2. Monogenic measures. Let $\Sigma$ be a $\sigma$-field of subsets of a set $\Omega$ and denote by $s(\Sigma)$ the linear lattice of $\Sigma$-measurable simple functions. The linear lattice $b a(\Sigma)$ of bounded real-valued finitely additive set functions on $\Sigma$ can be identified with $L_{b}(s(\Sigma), \mathbf{R})$ (cf. [5, Section 2]). Under this identification the set $c a(\Sigma)$ of real-valued measures on $\Sigma$ becomes a band in $L_{b}(s(\Sigma), \mathbf{R})$.

Let $\Sigma^{\prime}$ be a sub- $\sigma$-field of $\Sigma$. We say that $\mu \in c a(\Sigma)$ is monogenic (antimonogenic) with respect to $\Sigma^{\prime}$ if it is a monogenic (antimonogenic) operator with respect to $s\left(\Sigma^{\prime}\right)$ and $c a(\Sigma)$. It is easily seen that $\mu \in c a(\Sigma)$ is monogenic if and only if $|\mu| \Sigma^{\prime}|=| \mu|| \Sigma^{\prime}$ and there is no other $\nu \in c a(\Sigma)$ with this property which coincides with $\mu$ on $\Sigma^{\prime}$. It is also clear that $\mu \in c a_{+}(\Sigma)$ is monogenic if and only if for any $\nu \in c a_{+}(\Sigma)$ with $\nu\left|\Sigma^{\prime}=\mu\right| \Sigma^{\prime}$ we have $\nu=\mu$ (cf. Lemma 1). This shows that our definition generalizes (in two directions) the one given by Berberian [2, p. 231] and considered also by Johnson [4, §3].

Clearly $\mu \in c a_{+}(\Sigma)$ is monogenic whenever $\Sigma$ is contained in the completion of $\Sigma^{\prime}$ with respect to $\mu \mid \Sigma^{\prime}$. By a result of Koś-Marczewski [7, Theorem 4] this condition is also necessary if $\Sigma$ is generated by $\Sigma^{\prime} \cup\{A\}$, where $A \subset \Omega$. Hence, in view of a theorem of Bierlein [1, Corollary 3] this subsists if $A$ is replaced by an arbitrary family of disjoint subsets of $\Omega$. Due to a theorem of Varadarajan [13, Corollary to Lemma 2.2]; see also [1, Corollary 4], the same is true in case $\Omega$ is an analytic subset of a Polish space, $\Sigma$ is its Borel $\sigma$-field and $\Sigma^{\prime}$ is an arbitrary $\sigma$-generated sub- $\sigma$-field of $\Sigma$. However, if $\Sigma$ is "much larger" than $\Sigma$ ' it may happen that $\mu$ is monogenic even though $\mu \mid \Sigma^{\prime}$ is complete (see [10] or [11]).

As a special case of Theorem 1 we get

COROLlary $2 .^{3}$ The set of monogenic measures is a band in $c a(\Sigma)$.

The next corollary generalizes a result of Johnson [4, Theorem 3.3].

COROllary 3. Each $\mu \in c a(\Sigma)$ can be uniquely decomposed in the following way: $\mu=\mu_{1}+\mu_{2}$, where $\mu_{1}, \mu_{2} \in c a(\Sigma), \mu_{1}$ is monogenic and $\mu_{2}$ is antimonogenic. In particular, if $\mu \ll \nu$, where $\nu \in c a(\Sigma)$ is monogenic, then so is $\mu$.

Proof. As $c a(\Sigma)$ is a band in $b a(\Sigma)$ [6, Example 25.3], the first assertion is a special case of Corollary 1. The second assertion follows from this since $\mu_{2} \ll \nu$ and $\left|\mu_{2}\right| \wedge|\nu|=0$ imply $\mu_{2}=0$ (cf. [6, p. 144, (ii)]).

We shall illustrate Corollary 3 by two examples.

EXAmple 1. Let $\Sigma$ be the Borel $\sigma$-field of the real line and let $\Sigma^{\prime}$ be its sub- $\sigma$-field consisting of all countable sets and their complements. Then a measure on $\Sigma$ is

\footnotetext{
${ }^{3}$ Let us note that in the case of measures the essential implications (ii) $\Rightarrow$ (iii) and (ii) $\Rightarrow$ (iv) of Lemma 1 can also be proved with the help of the Radon-Nikodym theorem.
} 
monogenic (resp., antimonogenic) if and only if it is discrete (resp., continuous, i.e., vanishing on points). Thus the decomposition of Corollary 3 coincides in this case with the decomposition of a Borel measure into its discrete and continuous parts.

Before passing to the other example let us note that if $\Omega$ is an uncountable Borel subset of a Polish space and $\mu$ is a (nonzero) continuous universal measure on $\Omega$, then $\mu$ is not monogenic with respect to the Borel $\sigma$-field $B(\Omega)$ of $\Omega$. Indeed, assume $\mu$ is a probability measure (cf. Corollary 2 ). For $\Omega=[0,1]$ and $\mu$ being an extension of Lebesgue measure the assertion follows from the well-known fact that $\mu$ is not invariant. In general, $\mu \mid B(\Omega)$ is pointwise isomorphic to Lebesgue measure on $B([0,1])([8$, Theorem 4.1 (ii)], or $[12$, p. 327$])$, so a reduction to the previous case is possible.

EXAMPLE 2. Let $\Omega$ be the real line again, let $\nu_{0}$ be the completion of a continuous Borel measure on $\Omega$ and let $\Sigma$ denote the domain of $\nu_{0}$. Choose $\Sigma^{\prime}$ as the Borel $\sigma$-field of $\Omega$. It turns out then that in case $\mu \in c a(\Sigma)$ is continuous Corollary 3 yields the Lebesgue decomposition of $\mu$ relative to $\nu_{0}$. This is because a continuous measure $\nu \in c a(\Sigma)$ is monogenic if and only if $\nu \ll \nu_{0}$. One implication follows from Corollary 3. To prove the other assume that $\nu \ll \nu_{0}$ fails. Then there is $\Omega_{0} \in \Sigma^{\prime}$ with $\left|\nu_{0}\right|\left(\Omega_{0}\right)=0$ and $|\nu|\left(\Omega_{0}\right)>0$. It follows that $\nu$ restricted to $\Omega_{0} \cap \Sigma$ is a continuous universal measure on $\Omega_{0}$, and so it is not monogenic with respect to the Borel- $\sigma$-field of $\Omega_{0}\left(=\Omega_{0} \cap \Sigma\right)$ (see the passage following Example 1). Hence $\nu$ is not monogenic.

In the sequel we shall restrict attention to positive measures. From the previous results we shall only need the obvious assertion that a positive measure majorized by a monogenic measure is itself monogenic. The following is a generalization of a result of Johnson [2, Exercise 70.14].

TheOREM 2. Let, for $i=1,2, \Sigma_{i}$ and $\Sigma_{i}^{\prime}$ be $\sigma$-fields of subsets of a set $\Omega_{i}$ with $\Sigma_{i}^{\prime} \subset \Sigma_{i}$. Suppose $\mu_{i} \in c a_{+}(\Sigma)$ and $\mu \in c a_{+}\left(\Sigma_{1} \otimes \Sigma_{2}\right)$ is a product of $\mu_{1}$ and $\mu_{2}$.

(a) If $\mu_{i}$ is monogenic with respect to $\Sigma_{i}^{\prime}, i=1,2$, then $\mu$ is monogenic with respect to $\Sigma_{1}^{\prime} \otimes \Sigma_{2}^{\prime}$.

(b) If $\mu$ is the direct product of $\mu_{1}$ and $\mu_{2}$ and $\mu$ is monogenic with respect to $\Sigma_{1}^{\prime} \otimes \Sigma_{2}^{\prime}$, then $\mu_{i}$ is monogenic with respect to $\Sigma_{i}^{\prime}, i=1,2$.

Proof. Assertion (b) is obvious. To prove (a) suppose $\nu \in c a_{+}(\Sigma)$ and $\nu \mid \Sigma_{1}^{\prime} \otimes \Sigma_{2}^{\prime}$ $=\mu \mid \Sigma_{1}^{\prime} \otimes \Sigma_{2}^{\prime}$. It follows that $\nu\left(F_{1} \times F_{2}\right)=\mu\left(F_{1} \times F_{2}\right)<\mu_{1}\left(F_{1}\right)$ for $F_{i} \in \Sigma_{i}^{\prime}$. Fixing $F_{2}$ for a moment, we infer from the monogenicity of $\mu_{1}$ that $\nu\left(E_{1} \times F_{2}\right)=$ $\mu\left(E_{1} \times F_{2}\right)$ for all $E_{1} \in \Sigma_{1}$. A similar argument yields $\nu\left(E_{1} \times E_{2}\right)=\mu\left(E_{1} \times E_{2}\right)$ for all $E_{i} \in \Sigma_{i}^{\prime}$. Hence $\nu=\mu$.

Assertion (b) fails for nondirect products. Here is an example to this effect.

Example 3. By [9], (ii) there exist probability spaces $\left(\Omega_{i}, \Sigma_{i}^{\prime}, \mu_{i}^{\prime}\right), i=1,2$, a product $\mu^{\prime}$ of $\mu_{1}^{\prime}$ and $\mu_{2}^{\prime}$, and a set $A \subset \Omega_{1}$ such that its inner $\mu_{1}^{\prime}$-measure equals 0 while the inner $\mu^{\prime}$-measure of $A \times \Omega_{2}$ equals 1 . Let $\Sigma_{1}$ be the $\sigma$-field generated by $\Sigma_{1}^{\prime} \cup\{A\}$ and $\Sigma_{2}=\Sigma_{2}^{\prime}$. Then $\mu^{\prime}$ extends uniquely to a measure $\mu \in c a_{+}\left(\Sigma_{1} \otimes \Sigma_{2}\right)$ but $\mu_{1}^{\prime}$ can be extended in different ways to a positive measure on $\Sigma_{1}$. 
ADDED IN PROOF. The same example as in [10], [11] has also been given by $Z$. Riečanová, $A$ monogenic Baire measure need not be completion regular, Mat. Časopis Sloven. Akad. Vied 24 (1974), 275-276.

\section{REFERENCES}

1. A. Ascherl and J. Lehn, Two principles for extending probability measures, Manuscripta Math. 21 (1977), 43-50.

2. S. K. Berberian, Measure and integration, Wiley, New York, 1965.

3. G. Jameson, Ordered linear spaces, Lecture Notes in Math., vol. 141, Springer-Verlag, New York, 1970.

4. R. A. Johnson, Some types of Borel measure, Proc. Amer. Math. Soc. 22 (1969), 94-99.

5. Z. Lipecki, D. Plachky and W. Thomsen, Extensions of pasitive operators and extreme points. I, Colloq. Math. 42 (1979), 279-284.

6. W. A. J. Luxemburg and A. C. Zaanen, Riesz spaces. I, North-Holland, Amsterdam, 1971.

7. J. Koś and E. Marczewski, Extensions of measure, Fund. Math. 36 (1949), 267-276.

8. E. Marczewski (Szpilrajn), On absolutely measurable sets and functions, C. R. Soc. des Sciences et des Lettres de Varsovie 30 (1937), 39-67. (Polish, French summary)

9. E. Marczewski and C. Ryll-Nardzewski, Remarks on the compactness and nondirect products of measures, Fund. Math. 40 (1953), 165-170.

10. A. F. Martin, A note on monogenic Baire measures, Amer. Math. Monthly 84 (1977), 554-555.

11. D. Plachky, Extremal and monogenic additive set functions, Proc. Amer. Math. Soc. 54 (1976), 193-196.

12. H. L. Royden, Real analysis, London, 1970.

13. V. S. Varadarajan, Groups of automorphisms of Borel spaces, Trans. Amer. Math. Soc. 109 (1963), 191-220.

Institute of Mathematics, Polish Academy of Sciences, Kopbrnica 18, 51-617 Wrockaw, PoLAND

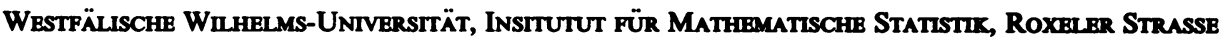
64, 4400 MÜnster, Federal RepUblic OF Germany 